\title{
The Challenging Evaluation of Patients with Severe Psoriasis for Latent Tuberculosis: An Important Indication for IGRA
}

\author{
Stamatis Katsenos ${ }^{*}, 1$, Melita Nikolopoulou ${ }^{1}$, Georgia Tsiouri ${ }^{2}$, \\ Ioannis D. Bassukas ${ }^{2}$ and Stavros H. Constantopoulos ${ }^{1}$ \\ ${ }^{I}$ Department of Pneumonology, University Hospital of Ioannina, Ioannina, Greece \\ ${ }^{2}$ Department of Skin and Venereal Diseases, University Hospital of Ioannina, Ioannina, Greece
}

\begin{abstract}
It is well-established that tumour necrosis factor (TNF)- $\alpha$-antagonist regimens are advisable for the control of moderate to severe psoriasis; however the application of these agents is associated with increased risk of TB reactivation. Screening for latent tuberculosis infection (LTBI) is indispensable prior to treatment inception in order to diminish the risk of active TB. Although tuberculin skin test (TST) still represents a useful tool for LTBI detection, it is difficult to be performed and read in patients with extensive psoriatic lesions. In this paper, we report the case of a 65-year-old male with severe psoriasis, who was evaluated by an interferon-gamma release assay (IGRA) for LTBI diagnosis prior to antiTNF- $\alpha$ therapy. Furthermore, the physiological aspects of interferon-gamma release assays are discussed emphasizing the value of these novel immunodiagnostic tests (IGRAs) for presumable LTBI in all patients with extensive skin disorders.
\end{abstract}

Keywords: Tuberculin skin test, interferon-gamma release assay, latent tuberculosis infection, psoriasis, anti-tumour necrosis factor therapy.

\section{INTRODUCTION}

Epidemiological evidence predicts that about one-third of the earth's population is presently infected by Mycobacterium tuberculosis, including a considerable fraction of individuals with latent tuberculosis infection (LTBI) [1]. Psoriasis with a worldwide prevalence of about $1 \%$ is a common chronic inflammatory skin disease with considerable impact on the quality of life of the affected individuals. Different systemic therapies, including TNF- $\alpha-$ antagonist regimens, are now available for the control of moderate to severe psoriasis; however the application of most of these modalities is connected with increased risk of TB reactivation [2]. Therefore, pre-screening of these patients for LTBI is mandatory prior to treatment initiation in order to minimize the risk of treatment-induced $\mathrm{TB}$ activation.

However, the diagnosis of LTBI is subjected to considerable clinical uncertainty. Tuberculin skin testing (TST) based guidelines are still widely applied to evaluate patients for LTBI. TST has been extensively used for nearly 100 years in clinical practice, yet it has several drawbacks: low specificity in patients with previous bacillus CalmetteGuérin (BCG) vaccination or exposure to non-tuberculous mycobacterial species as well as low sensitivity among patients with impaired immune function. Moreover in some patients, like those with severe psoriasis, extensive skin morbidity barely leaves healthy skin islands for TST measurement $[3,4]$. Although this is a rather uncommon

*Address correspondence to this author at the University of Ioannina, Medical School, Department of Pneumonology, 45110, Ioannina, Greece; Tel: +30-26510-07535; Fax: +30-2651-07051; E-mail: skatsenos@yahoo.gr event, it may prove a decisive hurdle for the proper evaluation of TST by the Mantoux method. We hereby present an alternative to rule out LTBI.

\section{CASE REPORT}

A 65-year-old male with late-onset psoriasis presented with severe sub-erythrodermic disease covering almost completely his integument (Psoriasis Area and Severity Index, PASI=29.8). During the last 5 years he was serially treated with topical corticosteroids and acitretin, cyclosporine and efalizumab with only partial improvement. Ongoing deterioration of his condition insisted treatment with infliximab. The patient had no history of $M$. tuberculosis exposure or other known risk factors for tuberculosis. A chest radiograph was carried out with normal findings. QuantiFERON-TB Gold in-Tube (QFT-GIT) assay was performed in our patient for the pretreatment assessment of his M. tuberculosis infection status showing negative results. QFT-GIT was preferred instead of the standard TSTbased methodology because of his extensive skin involvement. Inliximab was started as indicated with substantial improvement of his psoriasis (PASI 8.3 and 1.2 after 2 and 4 months of treatment respectively). No signs of systemic infections, including tuberculosis were recorded during the one-year follow-up period under infliximab treatment.

\section{DISCUSSION}

Recently in vitro immunodiagnostic assays have been developed in order to surmount some of the TST limitations. These assays measure interferon (IFN)- $\gamma$ production in whole blood or production of peripheral blood mononuclear cells (PBMC) in response to antigens (early secreted antigen; 
ESAT-6, culture filtrate protein; CFP-10) that are specific for $M$. tuberculosis and absent from BCG and most environmental mycobacteria. Herein, these assays will be referred to as IFN-gamma release assays (IGRA). The two commercially available tests are the T SPOT-TB assay, an enzyme-linked immunosorbent spot or ELISPOT test that uses peripheral blood mononuclear cells, and the QuantiFERON-TB Gold (QFT-G), an enzyme-linked immunosorbent assay (ELISA) that uses whole blood [5]. Recently, a novel in-tube format of QFT-G (QFT-GIT) has been marketed, which, apart from peptides of ESAT-6 and CFP-10, contains an additional peptide of the $M$. tuberculosis-specific antigen TB7.7 [6]. This newest version of QFT-G was employed in the present case.

Meanwhile, accumulating evidence suggests that commercially available interferon-gamma release assays (IGRAs) have higher specificity than the TST in detecting LTBI, particularly in BCG-vaccinated patients as well as greater sensitivity to detect infection after a known $M$. tuberculosis exposure in settings with low tuberculosis incidence [5,7]. IGRAs may be better at detecting recent rather than remote infection. It has been hypothesized that short incubation interferon-gamma release assays (both commercial assays use 16-24h incubation) detect responses of activated effector $\mathrm{T}$ cells that have recently encountered antigens in vivo and can therefore rapidly release interferon- $\gamma$ when stimulated in vitro. On the contrary, long-lived central memory $\mathrm{T}$ cells that may persist even after clearance of the organism (previously treated tuberculosis) may be less likely to release interferon- $\gamma$ during short incubation period [8]. Moreover, IGRAs seem to be superior to TST to detect LTBI in immunosuppressed patients [9].

Here on the occasion of such a patient with psoriatic erythrodermia, who had to be evaluated for LTBI prior to the initiation of anti-tumour necrosis factor-alpha (TNF- $\alpha$ ) therapy we would like to draw attention to the decisive advantage of IGRAs over the TST-based methodology in the evaluation of patients with extensive skin disease.

The assessment of the value of IGRAs as diagnostic tools in detecting LTBI in patients that are candidates for receiving anti-TNF- $\alpha$ therapy is currently under investigation [10]. Newly we showed that adherence to TST-based TB evaluation overestimates the risk of LTBI in adult patients with overt plaque psoriasis [4]. The present report highlights an additional hurdle in the proper evaluation of TST in the subset of dermatological patients and is a reminder that IGRAs should be considered as first-line diagnostic procedure for putative LTBI in all patients with extensive skin morbidity, like psoriatic erythrodermia, severe atopic eczema or Sezary syndrome, where the application of TST is unfeasible.

\section{ACKNOWLEDGEMENT AND CONFLICT OF INTEREST}

None declared.

\section{REFERENCES}

[1] World Health Organization. Global tuberculosis control: epidemiology, strategy, financing: WHO report 2009. Geneva, Switzerland: World Health Organization 2009. Available at http://www.who.int/tb/publications/global report/2009/pdf/report without_annexes.pdf Accessed June 16, 2010

[2] Doherty SD, Van Voorhees A, Lebwohl MG, et al. National Psoriasis Foundation: National psoriasis foundation consensus statement on screening for latent tuberculosis infection in patients with psoriasis treated with systemic and biologic agents. J Am Acad Dermatol 2008; 59: 209-17.

[3] Lee E, Holzman RS. Evolution and current use of the tuberculin test. Clin Infect Dis 2002; 34: 365-70.

[4] Tsiouri G, Gaitanis G, Kiorpelidou D, et al. Tuberculin skin test overestimates tuberculosis hypersensitivity in adult patients with psoriasis. Dermatology 2009; 219: 119-25.

[5] Pai M, Zwerling A, Menzies D. Systematic review: T-cell-based assays for the diagnosis of latent tuberculosis infection: an update. Ann Intern Med 2008; 149: 177-84.

[6] Cellestis Limited QuantiFERON-TB Gold (in-tube method) [package insert][on-line]. Available athttp://www.cellestis.com/IR $\mathrm{M} /$ content/aust/qtfproducts_tbgoldintube html [Accesses on: December 8, 2010].

[7] Mazurek GH, Jereb J, Vernon A, et al. IGRA Expert Committee, Centers for Disease Control and Prevention (CDC). Updated guidelines for using interferon gamma release assays to detect Mycobacterium tuberculosis infection- United States, 2010. MMWR Recomm Rep 2010; 59(RR-5): 1-25.

[8] Pai M, Dheda K, Cunningham J, Scano F, O’Brien R. T-cell assays for the diagnosis of latent tuberculosis infection: moving the research agenda forward. Lancet Infect Dis 2007; 7: 428-38.

[9] Kobashi Y, Mouri K, Obase Y, Fukuda M, Miyashita N, Oka M. Clinical evaluation of QuantiFERON TB-2G test for immunocompromised patients. Eur Respir J 2007; 30: 945-50.

[10] Balato N, Ayala F, Gaudiello F. Comparison of tuberculin skin test and interferon- $\gamma$ assays in patients with moderate to severe psoriasis who are candidates for antitumour necrosis factor- $\alpha$ therapy. Br J Dermatol 2008; 158: 847-8. 